\title{
Accurate differential analysis of transcription factor activity from gene expression
}

\author{
Viren Amin ${ }^{1,2}$, Murat Can Cobanoglu ${ }^{1, *}$ \\ ${ }^{1}$ Lyda Hill Department of Bioinformatics, UT Southwestern Medical Center, Dallas, TX \\ ${ }^{2}$ Current address: Baebies Inc, Durham, NC \\ ${ }^{*}$ Corresponding author; email: murat.cobanoglu@utsouthwestern.edu
}

\begin{abstract}
We present EPEE (Effector and Perturbation Estimation Engine), a method for differential analysis of transcription factor (TF) activity from gene expression data. EPEE addresses two principal challenges in the field, namely incorporating context-specific TF-gene regulatory networks, and accounting for the fact that TF activity inference is intrinsically coupled for all TFs that share targets. Our validations in well-studied immune and cancer contexts show that addressing the overlap challenge and using state-of-the-art regulatory networks enable EPEE to consistently produce accurate results. (Accessible at: https://github.com/Cobanoglu-Lab/EPEE)
\end{abstract}

\section{Main text}

Differential analysis of gene expression data is commonly used to dissect the mechanism of phenotypes of interest ${ }^{1}$. Commonly used differential expression (DE) methods ${ }^{2-5}$ do not account for the regulation of gene expression by transcription factors (TFs), even though TFs have a key role in controlling the transcriptome ${ }^{6}$. This shortcoming has prompted the development of differential regulation (DR) methods ${ }^{7-13}$, however these methods either do not permit the full representation of available context-specific regulatory data ${ }^{7,8,10-13}$ or interrogate regulators individually ${ }^{9,14}$ resulting in potential false positives for overlapping regulons ${ }^{8}$. To address these issues, we have developed the Effector and Perturbation Estimation Engine (EPEE) which infers the regulatory activity of all TFs jointly, constrained under the context-specific TF regulatory networks (Fig. 1).

EPEE models gene expression as the result of latent activity by TF gene products. We use the context-specific TF regulatory graph to prune inaccessible targets for each TF regulon, and then infer the activity of all TFs jointly with a single multivariate model (Supplementary Note 1). The activity of any TF over its target genes are inherently related with each other. We use graph constrained fused lasso ${ }^{15}$ to reflect that intuition. Briefly, fused lasso ${ }^{16}$ is a method for multivariate regression where the inference of model parameters are "fused" according to a priori established relationships. Graph constrained fused lasso ${ }^{15}$ is an extension to the setting where the inferred parameters are related with a graph structure. In EPEE, we fuse the parameters of the model to respect the context-specific regulatory network during TF activity inference. Crucially, since we represent the inference of all TF activity as a single (multivariate regression) problem, our inference automatically adjusts for overlapping TF regulons.

Overlap among TF regulons is widely prevalent, based on the state-of-the-art TF regulatory networks ${ }^{17}$ (Supplementary Fig. 1). Of the 206,403 TF-TF pairs in the CD4 ${ }^{+}$T cell context, 206,352 (99.9\%) overlap with each other to some degree (Supplementary Fig. 1c). To check if this is a feature specific to a few contexts, we evaluated all the 394 human contexts for which TF regulatory graphs are available ${ }^{17}$. We found that even the least complex context has overlaps among $96.4 \%$ of all the TF-TF pairs, whereas the median was $98 \%$ (Supplementary Fig. 1d). Causal inference theory dictates that all latent random variables that cause an effect 
become dependent when conditioned on the outcome ${ }^{18}$. Consequently, when inferring TF activity (latent cause) from gene expression (observed outcome), the activity inference for all TFs with overlapping regulons are dependent on each other. Hence, we argue that the latent TF activity inference must be solved jointly and we propose a single, multivariate model.

Most previous approaches on TF activity inference calculate each TF's activity individually ${ }^{7-14}$ and only aggregate at a later stage. For example, in the popular Gene Set Enrichment Analysis ${ }^{14}$ (GSEA), the running sum statistic evaluates each TF's activity individually. Likewise, MARINa ${ }^{8}$ or VIPER ${ }^{12}$ use mutual information to estimate TF activity, and this measure evaluates each TF separately. These two latter methods ${ }^{8,12}$ utilize a post hoc correction for this problem, called "shadow analysis", but its generality is unclear. To demonstrate the improvement that the joint TF activity model in EPEE provides over previous approaches, we conducted comparative evaluations.

We conducted comparative validation studies in two independent and well-studied contexts with known driver TFs: T helper cell differentiation and colorectal adenocarcinoma. The immune cell context enables us to compare methods within the context of normal transcriptional regulation. The cancer dataset, on the other hand, enables us to evaluate performance under the disrupted regulatory state that accompanies genomic instability in carcinogenesis ${ }^{19}$. Furthermore, the $\mathrm{T}$ cell data is homogeneous (i.e. highly purified biological replicates of the exact same cell types), while the cancer data from TCGA ${ }^{20}$ represents heterogeneous input because of both tumor purity $^{21}$ and subclonality ${ }^{22}$. The final major difference between the two datasets is the number of samples: the immune cell differentiation dataset has five samples per class (representative for a standard research laboratory effort), whereas the cancer dataset has close to five hundred samples (representative of a major consortium effort). In summary, we carefully selected these two validation studies to cover the diverse range of conditions in which differential TF activity assessments can be used.

Our first validation study was to identify the known driver TFs controlling CD4 ${ }^{+}$naïve $T$ cells differentiation to $T$ helper cells 1,2 , and $17\left(T_{h} 1, T_{h} 2, T_{h} 17\right)$. We selected the driver TFs as follows: STAT6 ${ }^{23-27}$ and GATA3 ${ }^{28}$ for $\mathrm{T}_{\mathrm{h}} 2$; TBX $21^{29}$, STAT $1^{30}, \mathrm{STAT4}^{30}$ for $\mathrm{T}_{\mathrm{h}} 1$; and RORa ${ }^{31}$, STAT3 $^{31,32}$, and ARID5A ${ }^{33}$ for $T_{h} 17$ differentiation of CD4 ${ }^{+}$T cells. We input the same RNA-seq data $^{34}$ to all methods (Supplementary Note 2). We quantified performance as the ranking of the ground truth TFs with each method, with higher rank signifying better performance. We used ten alternative methods that represent a variety of inference and regulation models (Supplementary Note 3). Eight were developed and/or previously used to estimate regulatory activity $^{7-14}$. We also included two differential expression methods to test the effectiveness for searching for an increase in the expression of the TF itself as a marker of its activity: ANOVA as a canonical differential expression analysis method $^{11}$, and sleuth as a state-of-the-art DE method $^{5}$. Of the eight differential regulation methods, three do not require an explicit regulatory network ${ }^{7,10,11}$ while the remaining five utilize a mathematical representation that evaluates each TF regulon individually. Among the alternatives, DISCERN ${ }^{11}$ is the only method that also solves the TF activity inference problem jointly for all TFs, albeit cannot incorporate a fully specified TFgene network. Therefore, we tested multiple members of each category of method a practitioner can use to identify TF activity. We observed that while other methods show significant variation and often fail to properly identify the known drivers, EPEE is consistently accurate in ranking ground truth TFs as differentially active (Fig. 2).

EPEE can incorporate context-specific TF regulatory networks with weighted edges, which are available thanks to recent large-scale data collection efforts such as ENCODE ${ }^{35}$ or FANTOM5 ${ }^{36}$ (Supplementary Table 2). Among the alternative methods, only REACTIN ${ }^{9}$ and i-score ${ }^{13}$ can 
utilize these rich regulatory graphs. GSEA uses $\mathrm{MSigDB}^{37} \mathrm{TF}$ target gene sets, that cannot represent TF-gene edge weights; MARINa and VIPER utilize ARACNE ${ }^{38}$ networks built using only transcriptomic data; while D-score ${ }^{7}, \mathrm{LNS}^{10}$, and DISCERN ${ }^{11}$ do not utilize any regulatory graph. The methods without networks serve as controls for the quality of the state-of-the-art regulatory networks: if the current networks are too deficient (not all TF motifs are available, for example) then the network-independent methods should perform better, and vice versa. For the methods that can utilize context-specific TF regulatory graphs, including our method, we input the $\mathrm{CD}^{+}{ }^{+}$cell network that Marbach and colleagues curated ${ }^{17}$ using FANTOM $5^{36}$ data. EPEE consistently outperformed methods with a range of network inputs, showing that EPEE provides an improved model to benefit from the state-of-the-art regulatory networks.

We then elucidated the contributions to our method's performance. The regularization provides consistency (Supplementary Fig. 2), while the context-dependent network contributes to the accuracy (Supplementary Fig. 3). EPEE can resolve TFs with high regulon overlap, while other methods cannot necessarily do so (Supplementary Fig. 4). Furthermore, the performance is not the result of parameter tuning. We used an entirely independent dataset of acute myeloid leukemia (AML) gene expression (microarray data) to determine the default hyperparameter values (Supplementary Fig. 5) and we simply used these default settings for all the results in our study. Likewise, for the competing methods we also used the default settings, unless suggested otherwise in the manual or documentation for that method (Supplementary Note 3).

To test whether EPEE can generalize to other biological domains, we applied EPEE to identify driver TFs from colorectal adenocarcinoma (COAD) gene expression data in TCGA (Fig. 3). In this context, we compared against msVIPER and GSEA due to their popularity, and ANOVA as the standard differential expression method. We used known oncogenes $M Y C^{20,39}$ and SOX $9^{20,40}$ as ground truth TFs that are differentially active in cancer. On the other end, we used KLF4 ${ }^{41-43}$ as a TF known to be differentially active in normal tissue, since KLF4 is commonly inactivated in cancer via diverse mechanisms such as miRNA silencing ${ }^{44,45}$. We used EPEE to identify both the statistically significantly perturbed genes and regulators (Benjamini-Hochberg FDR $<0.05$, based on permutation tests as described in Supplementary Note 1) and show the inferred TF-gene regulation changes (Fig. 3a). EPEE correctly identified the differential activity of all three TFs (Fig. 3b). GSEA failed to identify SOX9 as an oncogene, and KLF4 as differentially active in normal. msVIPER correctly identified KLF4 as differentially active in normal, but misplaced both MYC and SOX9 by inferring many other TFs to be more active in cancer. Overall, EPEE outperformed alternative methods in cancer as well as it did in immune cells.

Finally, we used EPEE to infer differential TF activity separately for each consensus molecular subtype (CMS) of colorectal adenocarcinoma (Supplementary Fig. 6). We found that MYC, SOX9 and KLF4 activity were homogenous across each CMS subtype despite varying purity estimates in each CMS (Supplementary Fig. 7). We also discovered subtype-specific TFs with differential activity, some of which were reported to be colorectal adenocarcinoma related $\left(A S C L 2^{46-49}, E T V 4^{50}, H S F 1^{51}\right.$ ) while others represent novel predictions that can be tested by follow-up experimental work.

In conclusion, we assert that the prevalent overlap among TF regulons causes problematic TF activity inference by readily available existing methods and thus present a novel solution that models all TF activity as a single multivariate regression problem. We demonstrate consistently accurate results on well-studied immune and cancer contexts. We provide our method EPEE open source and freely available. 
bioRxiv preprint doi: https://doi.org/10.1101/296210; this version posted April 6, 2018. The copyright holder for this preprint (which was not

\section{Acknowledgements}

We would like to acknowledge Curtis Thorne, Didem Ağaç, David Farrar, Anne Satterthwaite, Maxim Grechkin, and Daniel Marbach for their helpful discussions.

\section{Funding}

We are supported by the UT Southwestern Distinguished Fellow startup funds awarded by the Lyda Hill Department of Bioinformatics. 


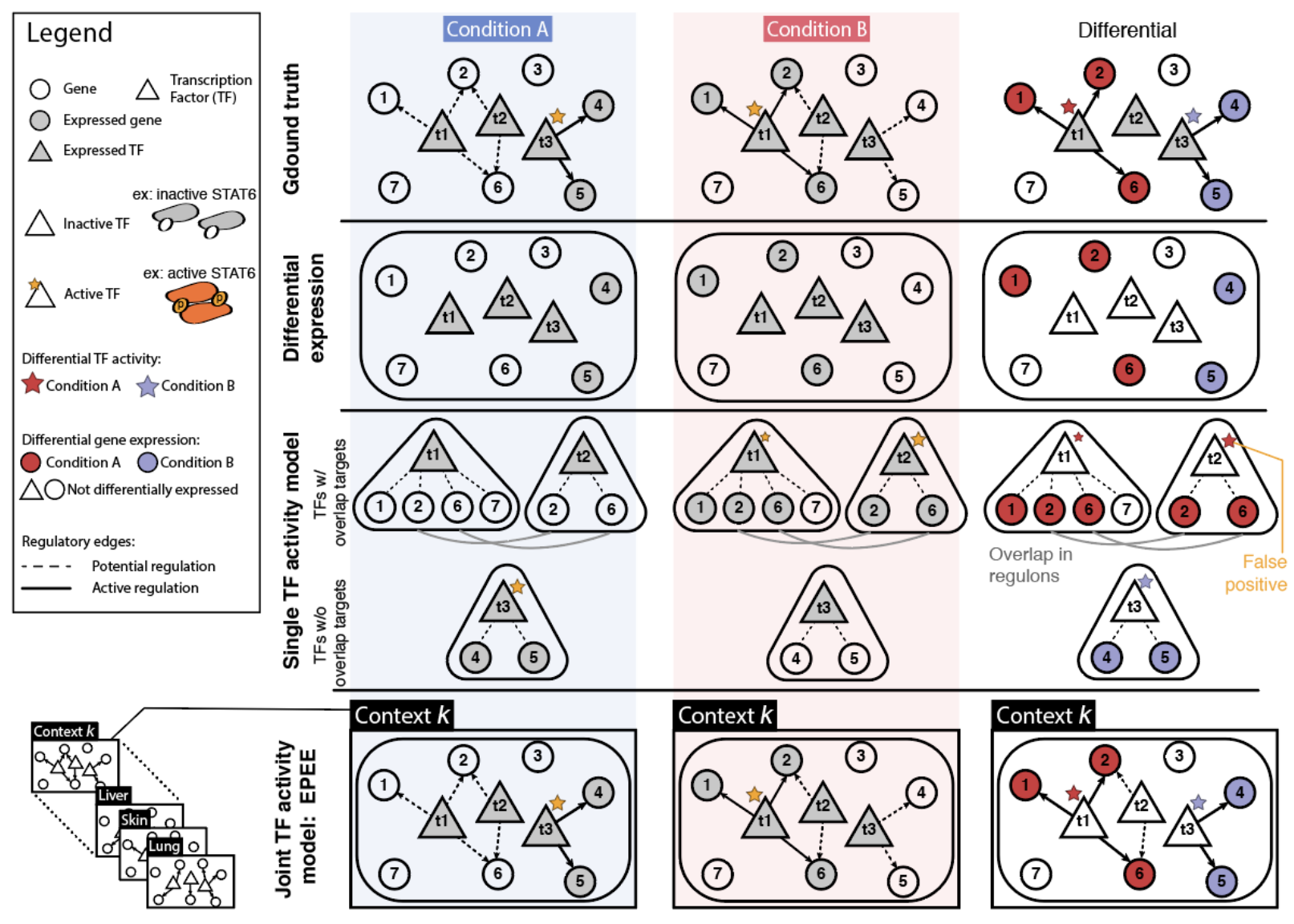

Figure 1. Schematic overview of EPEE in relation to alternative approaches.

We construct a schematic example to illustrate our motivation for developing EPEE. In this schematic, the ground truth (top row) is that one TF is active in each condition. Differential expression methods (second row) do not account for any regulatory relationship among genes, and simply report the genes with different expression. Single TF activity model based differential regulation methods (third row) evaluate each TF individually, and this is problematic when TF regulons overlap, leading to false positives. EPEE (bottom row) uses the appropriate contextspecific TF-gene regulatory network and models all TF activity as a single multivariate regression problem, to address both context-specific changes and overlapping regulons. 

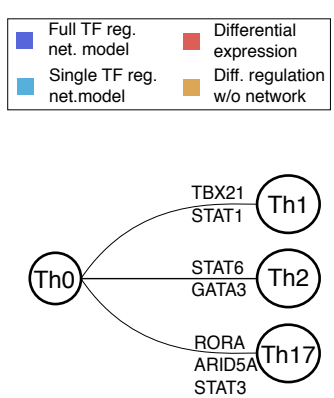

Th2

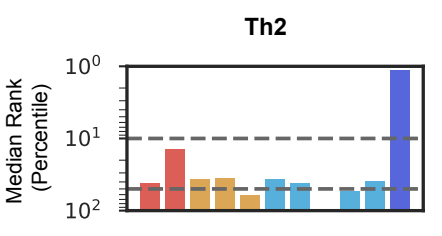

+ STAT6 q GATA3

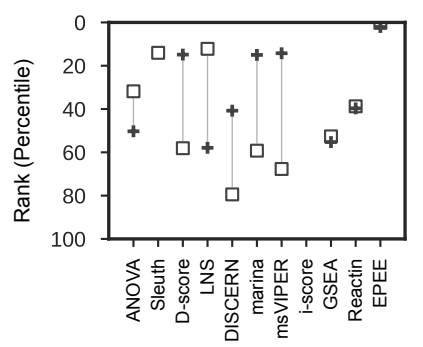

Th17

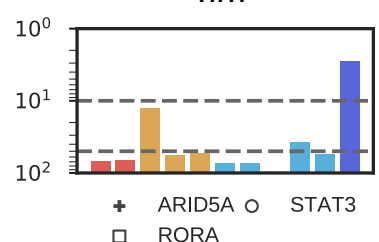

口 RORA

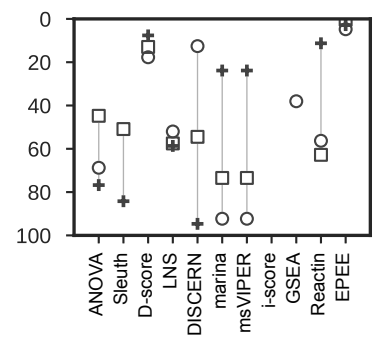

Th1
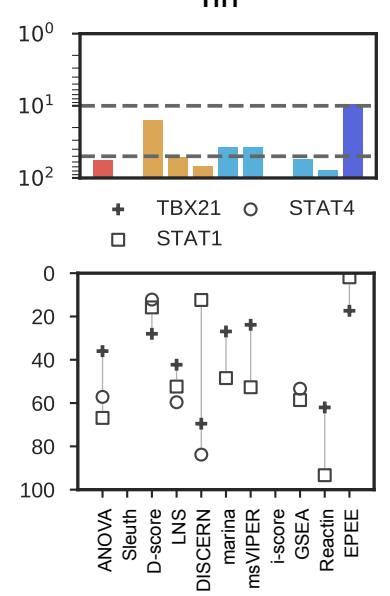

Figure 2: EPEE infers transcriptional regulators reliably and effectively. We tested regulator inference in CD4 naïve T cell differentiation to T helper $1\left(T_{h} 1\right), T_{h} 2, T_{h} 17$ cells. Drivers are known for each pathway therefore we can compare performance. EPEE performs remarkably better than all other DE (red) and DR (gold: no network, light blue: transcriptomic data driven network, dark blue: full regulatory network) methods. In the log percentile plots, the top dashed line represents the 90th percentile threshold, the bottom dashed line represents the 50the percentile. 


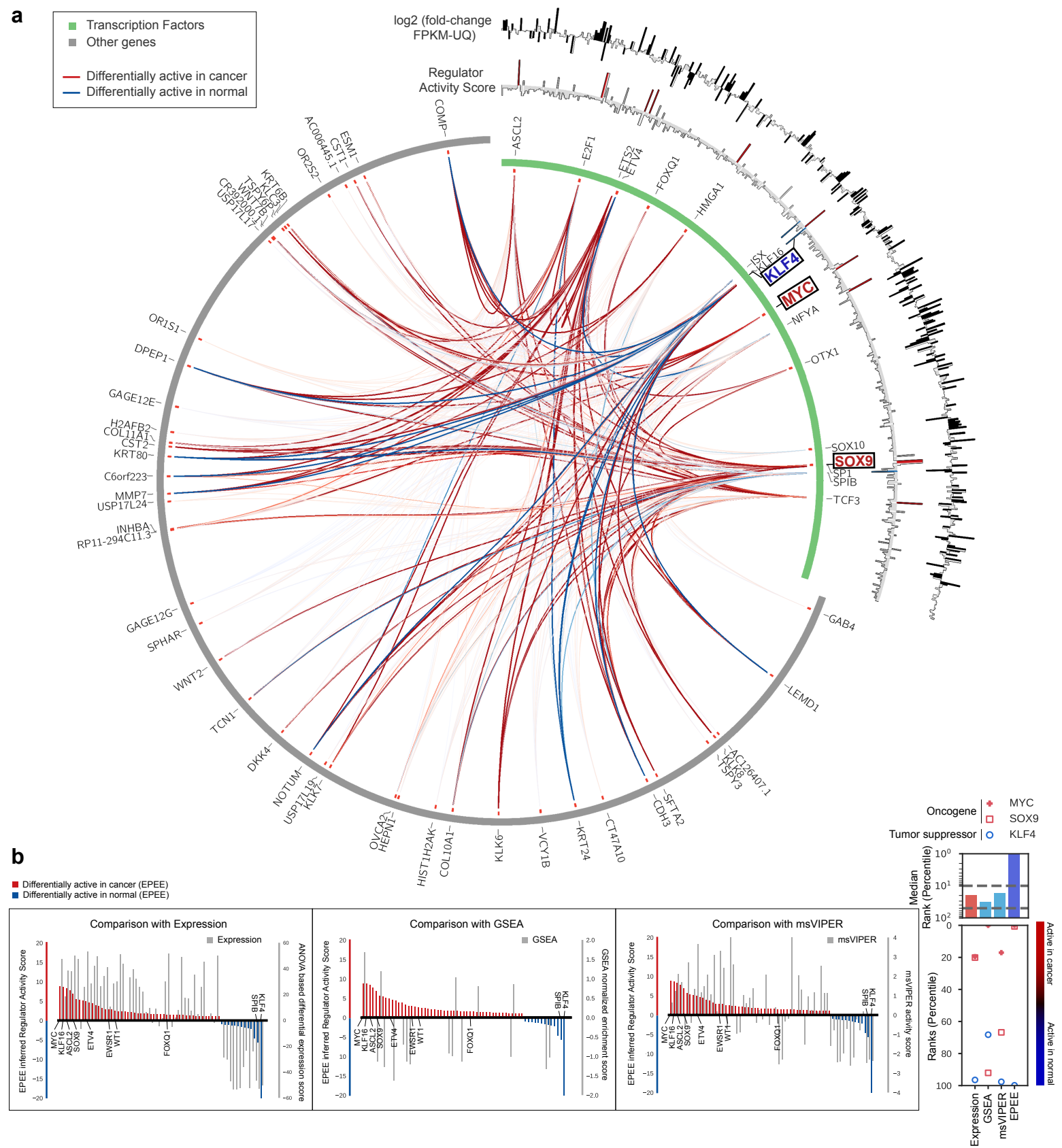

Figure 3: EPEE can accurately infer differential regulation events in cancer. (a) We conducted differential analysis of TF activity between colorectal adenocarcinoma (COAD) samples and tissue-matched controls using TCGA data. Circos plot shows differential regulation with red and blue TF-gene edges having high activity in cancer and normal. Green band maps TFs and grey band maps perturbed genes. (b) We also performed the same analysis using GSEA, msVIPER, and ANOVA. EPEE accurately identified the oncogenes MYC and SOX9 as differentially active, ranking MYC as the most differentially active TF in cancer. EPEE also identified tumor suppressor KLF4 to be differentially active in normal. 


\section{References}

1. Rapaport, F. et al. Comprehensive evaluation of differential gene expression analysis methods for RNA-seq data. Genome Biol. 14, R95 (2013).

2. Leek, J. T., Monsen, E., Dabney, A. R. \& Storey, J. D. EDGE: extraction and analysis of differential gene expression. Bioinformatics 22, 507-508 (2006).

3. Trapnell, C. et al. Differential analysis of gene regulation at transcript resolution with RNAseq. Nat. Biotechnol. 31, 46-53 (2013).

4. Anders, S. \& Huber, W. Differential expression analysis for sequence count data. Genome Biol. 11, R106 (2010).

5. Pimentel, H., Bray, N. L., Puente, S., Melsted, P. \& Pachter, L. Differential analysis of RNAseq incorporating quantification uncertainty. Nat. Methods 14, 687-690 (2017).

6. Spitz, F. \& Furlong, E. E. M. Transcription factors: from enhancer binding to developmental control. Nat. Rev. Genet. 13, 613-626 (2012).

7. Wang, K. et al. Meta-analysis of inter-species liver co-expression networks elucidates traits associated with common human diseases. PLoS Comput. Biol. 5, e1000616 (2009).

8. Lefebvre, C. et al. A human B-cell interactome identifies MYB and FOXM1 as master regulators of proliferation in germinal centers. Mol. Syst. Biol. 6, 377 (2010).

9. Zhu, M., Liu, C.-C. \& Cheng, C. REACTIN: regulatory activity inference of transcription factors underlying human diseases with application to breast cancer. BMC Genomics 14, 504 (2013).

10. Guan, Y., Dunham, M. J., Troyanskaya, O. G. \& Caudy, A. A. Comparative gene expression between two yeast species. BMC Genomics 14, 33 (2013).

11. Grechkin, M., Logsdon, B. A., Gentles, A. J. \& Lee, S.-I. Identifying Network Perturbation in Cancer. PLoS Comput. Biol. 12, e1004888 (2016).

12. Alvarez, M. J. et al. Functional characterization of somatic mutations in cancer using network-based inference of protein activity. Nat. Genet. 48, 838-847 (2016). 
13. Berchtold, E., Csaba, G. \& Zimmer, R. Evaluating Transcription Factor Activity Changes by Scoring Unexplained Target Genes in Expression Data. PLoS One 11, e0164513 (2016).

14. Subramanian, A. et al. Gene set enrichment analysis: a knowledge-based approach for interpreting genome-wide expression profiles. Proc. Natl. Acad. Sci. U. S. A. 102, 1554515550 (2005).

15. Kim, S. \& Xing, E. P. Statistical estimation of correlated genome associations to a quantitative trait network. PLoS Genet. 5, e1000587 (2009).

16. Tibshirani, R., Saunders, M., Rosset, S., Zhu, J. \& Knight, K. Sparsity and smoothness via the fused lasso. J. R. Stat. Soc. Series B Stat. Methodol. 67, 91-108 (2005).

17. Marbach, D. et al. Tissue-specific regulatory circuits reveal variable modular perturbations across complex diseases. Nat. Methods 13, 366-370 (2016).

18. Pearl, J. Causal inference in statistics: An overview. Stat. Surv. 3, 96-146 (2009).

19. Tabassum, D. P. \& Polyak, K. Tumorigenesis: it takes a village. Nat. Rev. Cancer 15, 473483 (2015).

20. The Cancer Genome Atlas Network. Comprehensive Molecular Characterization of Human Colon and Rectal Cancer. Nature 487, 330-337 (2012).

21. Aran, D., Sirota, M. \& Butte, A. J. Systematic pan-cancer analysis of tumour purity. Nat. Commun. 6, 8971 (2015).

22. Guinney, J. et al. The consensus molecular subtypes of colorectal cancer. Nat. Med. 21, 1350-1356 (2015).

23. Elo, L. L. et al. Genome-wide profiling of interleukin-4 and STAT6 transcription factor regulation of human Th2 cell programming. Immunity 32, 852-862 (2010).

24. Kaplan, M. H., Schindler, U., Smiley, S. T. \& Grusby, M. J. Stat6 Is Required for Mediating Responses to IL-4 and for the Development of Th2 Cells. Immunity 4, 313-319 (1996).

25. Shimoda, K. et al. Lack of IL-4-induced Th2 response and IgE class switching in mice with disrupted Stat6 gene. Nature 380, 630-633 (1996). 
26. Takeda, K. et al. Essential role of Stat6 in IL-4 signalling. Nature 380, 627-630 (1996).

27. Zhu, J., Guo, L., Watson, C. J., Hu-Li, J. \& Paul, W. E. Stat6 is necessary and sufficient for IL-4's role in Th2 differentiation and cell expansion. J. Immunol. 166, 7276-7281 (2001).

28. Ouyang, W. et al. Inhibition of Th1 development mediated by GATA-3 through an IL-4independent mechanism. Immunity 9, 745-755 (1998).

29. Szabo, S. J. et al. A novel transcription factor, T-bet, directs Th1 lineage commitment. Cell $100,655-669(2000)$.

30. Oh, H. \& Ghosh, S. NF-kB: roles and regulation in different CD4(+) T-cell subsets. Immunol. Rev. 252, 41-51 (2013).

31. Yang, X. O. et al. T helper 17 lineage differentiation is programmed by orphan nuclear receptors ROR alpha and ROR gamma. Immunity 28, 29-39 (2008).

32. Luckheeram, R. V., Zhou, R., Verma, A. D. \& Xia, B. CD4+ T Cells: Differentiation and Functions. Journal of Immunology Research 2012, (2012).

33. Masuda, K. et al. Arid5a regulates naive CD4+ T cell fate through selective stabilization of Stat3 mRNA. J. Exp. Med. 213, 605-619 (2016).

34. Bonnal, R. J. P. et al. De novo transcriptome profiling of highly purified human lymphocytes primary cells. Scientific Data 2, sdata201551 (2015).

35. Kheradpour, P. \& Kellis, M. Systematic discovery and characterization of regulatory motifs in ENCODE TF binding experiments. Nucleic Acids Res. 42, 2976-2987 (2014).

36. FANTOM Consortium and the RIKEN PMI and CLST (DGT) et al. A promoter-level mammalian expression atlas. Nature 507, 462-470 (2014).

37. Liberzon, A. et al. The Molecular Signatures Database (MSigDB) hallmark gene set collection. Cell Syst 1, 417-425 (2015).

38. Margolin, A. A. et al. ARACNE: an algorithm for the reconstruction of gene regulatory networks in a mammalian cellular context. BMC Bioinformatics 7 Suppl 1, S7 (2006).

39. Wiegering, A. et al. Targeting Translation Initiation Bypasses Signaling Crosstalk 
Mechanisms That Maintain High MYC Levels in Colorectal Cancer. Cancer Discov. 5, 768781 (2015).

40. Matheu, A. et al. Oncogenicity of the developmental transcription factor Sox9. Cancer Res. 72, 1301-1315 (2012).

41. Dang, D. T. et al. Overexpression of Krüppel-like factor 4 in the human colon cancer cell line RKO leads to reduced tumorigenecity. Oncogene 22, 3424-3430 (2003).

42. Zhao, W. et al. Identification of Krüppel-like factor 4 as a potential tumor suppressor gene in colorectal cancer. Oncogene 23, 395-402 (2004).

43. Ton-That, H., Kaestner, K. H., Shields, J. M., Mahatanankoon, C. S. \& Yang, V. W. Expression of the gut-enriched Krüppel-like factor gene during development and intestinal tumorigenesis. FEBS Lett. 419, 239-243 (1997).

44. Lv, H. et al. MicroRNA-92a Promotes Colorectal Cancer Cell Growth and Migration by Inhibiting KLF4. Oncol. Res. 23, 283-290 (2016).

45. Tang, W. et al. MicroRNA-29a promotes colorectal cancer metastasis by regulating matrix metalloproteinase 2 and E-cadherin via KLF4. Br. J. Cancer 110, 450-458 (2014).

46. Stange, D. E. et al. Expression of an ASCL2 related stem cell signature and IGF2 in colorectal cancer liver metastases with 11p15.5 gain. Gut 59, 1236-1244 (2010).

47. Jubb, A. M. et al. Achaete-scute like 2 (ascl2) is a target of Wnt signalling and is upregulated in intestinal neoplasia. Oncogene 25, 3445-3457 (2006).

48. Jubb, A. M., Hoeflich, K. P., Haverty, P. M., Wang, J. \& Koeppen, H. Ascl2 and 11p15.5 amplification in colorectal cancer. Gut 60, 1606-7; author reply 1607 (2011).

49. Zhu, R. et al. Ascl2 knockdown results in tumor growth arrest by miRNA-302b-related inhibition of colon cancer progenitor cells. PLoS One 7, e32170 (2012).

50. Moss, A. C. et al. ETV4 and Myeov knockdown impairs colon cancer cell line proliferation and invasion. Biochem. Biophys. Res. Commun. 345, 216-221 (2006).

51. Cen, H., Zheng, S., Fang, Y.-M., Tang, X.-P. \& Dong, Q. Induction of HSF1 expression is 
bioRxiv preprint doi: https://doi.org/10.1101/296210; this version posted April 6, 2018. The copyright holder for this preprint (which was not certified by peer review) is the author/funder, who has granted bioRxiv a license to display the preprint in perpetuity. It is made available under aCC-BY-ND 4.0 International license.

associated with sporadic colorectal cancer. World J. Gastroenterol. 10, 3122-3126 (2004). 